\title{
Electro Optic Modulation In a Polymer Ringresonator
}

\author{
A. Leinse, A. Driessen and M.B.J. Diemeer \\ Integrated Optical Micro Systems, University of Twente, P.O. Box 217, 7500 AE Enschede, The \\ Netherlands
}

\begin{abstract}
A thermo optic and electro optic (EO) tunable polymer ringresonator was realized and tested. The device consisted of a microring resonator made of the 4-dimethylamino-4 nitrostilbene (DANS) containing polymer and measurements were done on the through port of this device. The ring was used in a vertical coupling structure. The port waveguides were made of the photo-definable epoxy (SU8). The rings used had a diameter of $100 \mu \mathrm{m}$ and thermo optic tuning of about $170 \mathrm{pm} /{ }^{\circ} \mathrm{C}$ was measured. EO modulation was measured for TE polarization.
\end{abstract}

\section{REALIZATION}

A polymer micro-ringresonator was realized. The device realized is given in figure 1a. A schematic cross section (along the dotted line) of the vertically coupled device is given in figure 1b. By making the waveguide in the photo-definable SU8, a waveguide is created without an etching step in the fabrication process. Smooth rings were fabricated by reflowing

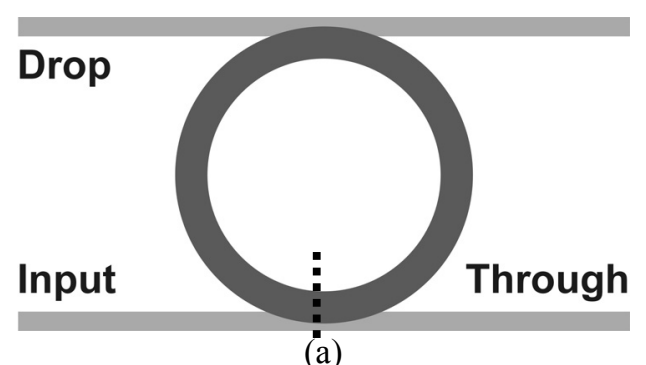

FIGURE 1 (a) Topview of the realized device.

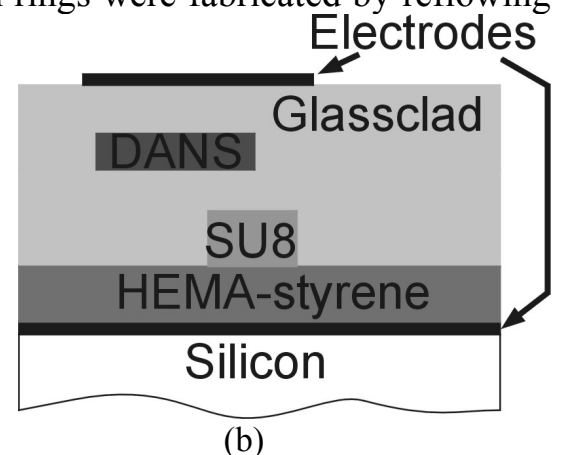

(b)

the DANS containing EO polymer ring after Reactive Ion Etching. When the sample is heated above its glass transition temperature, the ring starts to reflow and surface tension will smoothen the edge of the ringresonator (see figure $2 \mathrm{a}$ and $\mathrm{b}$ ). The electrodes that apply the electric modulation field over the ring are also used to pole the ring.

\section{CHARACTERIZATION}

An Erbium Doped Fiber Amplifier (EDFA) spectrum of a single polarization is coupled in with a polarization maintaining fiber at the input of a device with a $100 \mu \mathrm{m}$ ring. The signal of the through port is measured with a standard telecom fiber connected to a spectrum analyzer. Resonator dips of about $10 \mathrm{~dB}$ are observed and tunability of these dips is shown by heating up the resonator. A shift in the resonance dips of about $170 \mathrm{pm} /{ }^{\circ} \mathrm{C}$ is observed (figure 3 ). 


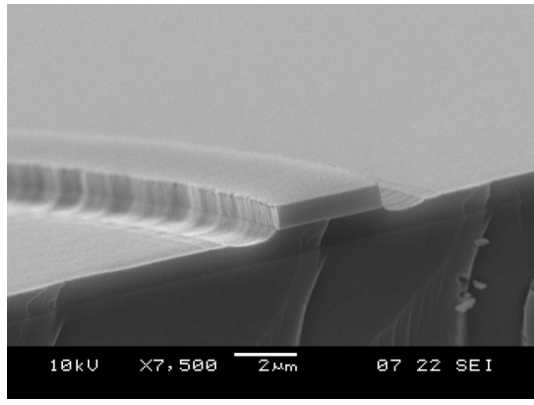

(a)

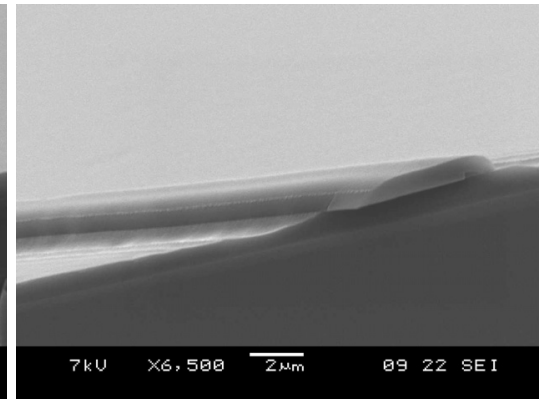

(b)

FIGURE 2 (a) Ringresonator before heating. (b) Ringresonator after heating and reflowing

Electro-Optic (EO) tunability is demonstrated by using a tunable laser instead of the EDFA and apply a $20 \mathrm{Vpp}$ sine modulation voltage $(+10$ to $-10 \mathrm{~V})$ over the electrodes. The signal from the through-port is measured with a lock-in amplifier and DC detector. Both results are given in figure $3 \mathrm{~b}$. To exclude the possibility of stress induced refractive index changes, the response was analyzed at the modulation frequency and at the double frequency. Because the change in refractive index caused by stress is independent of the field polarity, the effect can only be measured at the double modulation frequency. This measurement yielded close to zero-signal over the entire wavelength range.

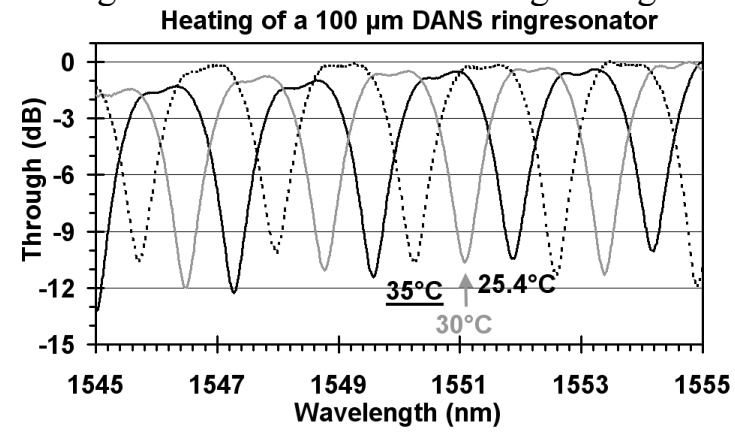

(a)

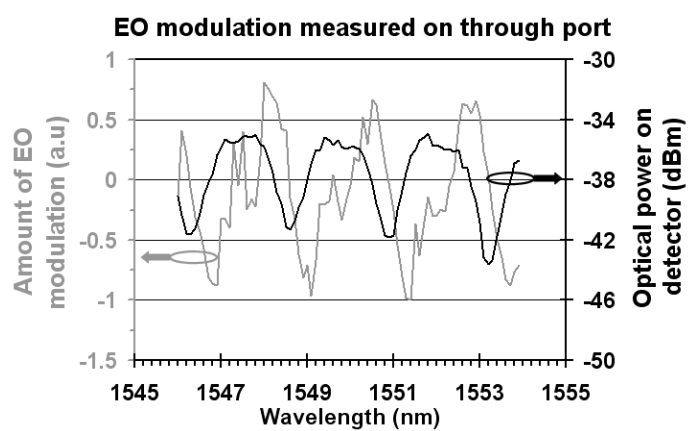

(b)

FIGURE 3 (a) Thermo optic tuning of the ringresonator. (b) Measurement (at the modulation frequency) of EO modulation on single ring resonator.

\section{CONCLUSIONS}

A polymer microring resonator was realized and characterized. Ringresonators with low scattering sidewalls can be fabricated by reflowing. Thermo optic tunability of $170 \mathrm{pm} /{ }^{\circ} \mathrm{C}$ and EO tunability were shown.

\section{REFERENCES}

[1] D.J.W. Klunder, E. Krioukov, F.S. Tan, T. van der Veen, H.F. Bulthuis, G. Sengo, C. Otto, H.J.W.M. Hoekstra, A. Driessen, "Vertically and laterally waveguide-coupled cylindrical microresonators in $\mathrm{Si}_{3} \mathrm{~N}_{4}$ on $\mathrm{SiO}_{2}$ technology", Appl. Phys. B., vol 73, 603-608, 2001

[2] Payam Rabiei, Willian H. Steier, Cheng Zhang, Larry Dalton, "Polymer Micro-Ring Filters and Modulators",Journal of Lightwave Technology, vol 20, no 11, November 2002.

[3] Brent E. Little, Sai T. Chu, "Theory of polarization rotation and conversion in vertically coupled microresonators", IEEE Photonics Technology Letters, vol 12, no 4, april 2000. 